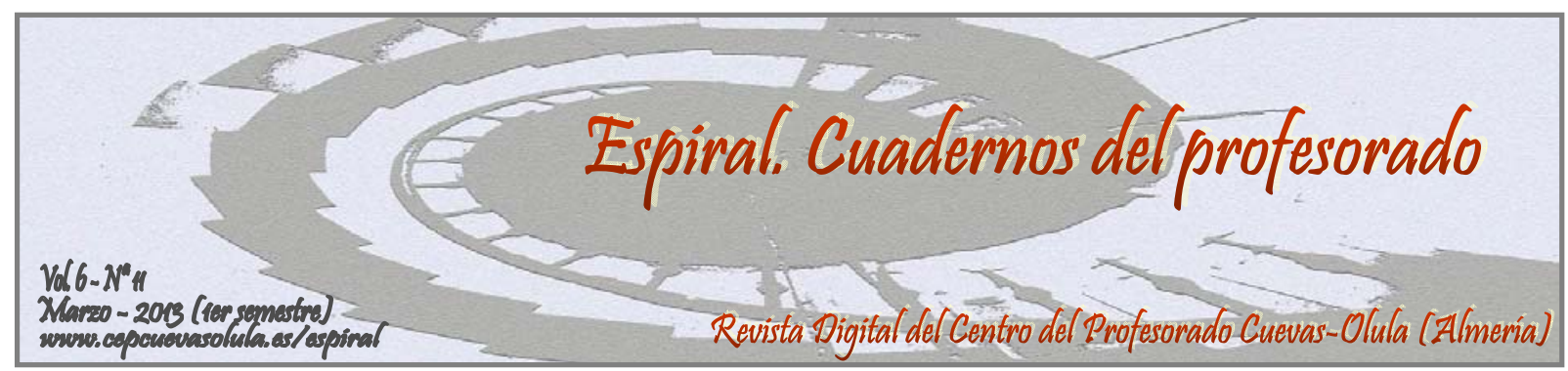

\title{
LA UTILIZACIÓN DEL MICROBLOGGING Y DE TWITTER COMO HERRAMIENTA DE ENSEÑANZA-APRENDIZAJE
}

\author{
MICROBLOGGING AND TWITTER USED AS A TOOL FOR LEARNING
}

\section{Fernando Checa García}

\author{
Facultad de Educación, Universidad Autónoma de Bacaramanga, Colombia
}

RESUMEN: Las nuevas herramientas generadas en el ámbito de la Web social están creando vías de apoyo a la creación de conocimiento mediante el uso de tecnologías sencillas y de cómodo uso. El objetivo de este artículo es mostrar como el uso del microblogging y de Twitter particularmente, puede ser una herramienta tecno-social que mejore los procesos de enseñanza-aprendizaje. Comenzando con el análisis del funcionamiento de la plataforma, se analiza en profundidad Twitter tanto desde el punto de vista de la generación de contenidos y su puesta en común, como desde la óptica de las interacciones sociales que se producen en él. Así mismo se han estudiado las posibilidades y ventajas que puede ofrecer su uso desde la escuela, tanto como proceso comunicativo entre docentes y discentes, así como desde la nueva construcción social virtualizada que genera y se aportan las posibles dificultades y problemas que pueden encontrarse los docentes o los alumnos en su uso. Twitter ofrece ventajas que van desde el establecimiento de sistemas de aprendizaje informal hasta la mejora de habilidades comunicativas y sociales, pero su uso no está exento de aspectos negativos, especialmente cuando no existe una estrategia previa de implantación en el aula para utilizarlo como refuerzo de las acciones de enseñanza y aprendizaje.

Palabras clave: Microblogging, Twitter, Redes Sociales, TIC

ABSTRACT: The new tools developed within the framework of the social web are creating ways of supporting the creation of knowledge through the use of simple technologies and comfortable use. The aim of this paper is to show how the use of microblogging and Twitter in particular, can be a tool to improve technological and social processes of teaching and learning. Starting with the analysis of the functioning of the platform, Twitter is analyzed in depth both in terms of content generation and sharing, as from the perspective of social interactions that occurs in it. We have also studied the possibilities and potential benefits from school use, as well as the communication process between teachers and students, and the new virtualized social construction generated, and we show the potential pitfalls and problems that teachers and students may find in their use. Twitter offers advantages, from the establishment of informal learning to communication and social skills improvement, but their use is not without negative aspects, especially when there is no previous strategy implementation in the classroom for its use as reinforcing teaching and learning activities.

Key words: Microblogging, Twitter, Social Network, ICT

Checa García, F. (2013). La utilización del microblogging y de twitter como herramienta de enseñanza-aprendizaje. Espiral. Cuadernos del Profesorado, 6(11), 19-27. Disponible en: http://www.cepcuevasolula.es/espiral.

Fecha de recepción: 12/03/2012

Fecha de aceptación: 30/06/2012
Enviar correspondencia a:

fcheca@unab.edu.com 


\section{1.- INTRODUCCIÓN. EL MICROBLOGGING Y SU KILLER app, Twitter}

Comparado con el uso clásico de los blogs, el microblogging supone acelerar la comunicación que se produce en la Web 2.0. Los posts tan breves requieren muy poco tiempo para su construcción. Son rápidas reflexiones o pensamientos publicados de forma inmediata. Las actualizaciones, frente al mayor tiempo que puede pasar en un blog, donde no es demasiado habitual que haya más de un post diario y habitualmente incluso menos, son mucho mayores. El usuario de los sistemas de microblogging suele actualizar varias veces al día, gracias a la facilidad que supone compartir de forma casi inmediata cualquier idea, sin apenas tener que preocuparse por la edición del post, el diseño o incluso la temática del mismo.

El microblogging es un fenómeno recientemente novedoso en el que el usuario realiza actualizaciones que suelen estar por debajo de los 200 caracteres (140 en el caso de Twitter, la aplicación de microblogging más usada) acerca de cualquier aspecto que resulte de interés para el usuario, a través de mensajes SMS, correo electrónico o vía aplicaciones Web. A principios de 2006 se lanzó Twitter y su forma de uso ha acabado por convertirse en punto de referencia del microblogging (Codel, 2006; Glaser, 2007). Su adopción temprana en Estados Unidos y su uso por parte de personalidades famosas así como la incorporación en su momento a la campaña presidencial norteamericana le ha convertido en la aplicación de microblogging más usada, pese a existir otras similares.

Twitter originalmente era una herramienta que permitía a los usuarios publicar en Internet micropost de un máximo de 140 caracteres. Contestando a la pregunta ¿Qué estás haciendo? Los usuarios registrados podían lanzar mensajes de todo aquello que les pasara por la cabeza. De una forma rápida y sencilla, sin que la personalización del entorno tuviera apenas importancia, Twitter se convertía en una opción muy utilizada que complementaba los blogs perfectamente. Lo que comenzó como una aplicación de "bajo impacto" se ha convertido actualmente en uno de los servicios de la Web Social más utilizados y de mayor crecimiento (Codel, 2006; Krisnamurty, Gill y Arlitt, 2008; Cheng y Evans, 2009) alcanzando a comienzos del año 2012 la cifra de 500 millones de usuarios.

Los usuarios de Twitter pueden actualizar su estado mediante la aplicación web, el uso de clientes de escritorio o para teléfonos móviles o a través del envío de mensajes vía SMS. Y sus perfiles pueden estar abiertos, de forma que cualquiera pueda acceder a ellos o mantenerlos privados, sólo para su red de contactos, denominados followers. Cuando un miembro de Twitter encuentra a otro cuyos mensajes le resultan interesantes o tan sólo quiere seguirle, le añade como amigo, enviándose automáticamente un correo electrónico de aviso, de forma que el nuevo contacto tiene la opción de bloquear al nuevo follower, si bien por defecto la aceptación se produce (algo diferente a otras redes sociales que exigen la aceptación expresa por parte del receptor).

En el caso de que el usuario tenga un perfil público, sus actualizaciones aparecen en el Public Timeline, una metafórica línea temporal donde un usuario puede ver las actualizaciones de aquellos a los que está siguiendo, independientemente de que ellos le sigan a él a su vez. Twitter permite a un usuario seguir las actualizaciones de otro miembro de la comunidad que haya sido añadido como "amigo". Un usuario que no es "amigo" de otro pero sigue sus actualizaciones es conocido genéricamente como follower, así la "amistad" puede entenderse de un modo recíproco o sólo desde una persona hacia otra.

Twitter ofrece múltiples formas de uso, según cada usuario decide utilizar la herramienta (Java, Finim, Song y Tseng, 2007). Al igual que ocurre con muchas aplicaciones Web 2.0, es la comunidad de usuarios la que decide cuál es la forma más interesante de aprovechar las características ofrecidas. A la respuesta inicial de la pregunta ¿Qué estás haciendo? los usuarios responden de diferentes formas (Mischaud, 2007; Milstein, Choudhury, Hochmuth, Lorica y Magoulas, 2008; Ebner y Schiefner 2008; Ebner y Maurer, 2009):

- Diario del momento. Los escritos se refieren a la rutina diaria o muestran lo que el usuario hace en el momento en el que los escribe. Es el primer y más extendido de los usos de Twitter. 
- Conversaciones. Los usuarios pueden realizar comentarios o réplicas a los mensajes de otros usuarios utilizando el símbolo @ seguido del nombre, apodo o nick de la persona replicada.

- Información compartida. Muchos usuarios utilizan Twitter para compartir con sus followers direcciones de Internet que consideran interesantes. Dadas las limitaciones de espacio a 140 caracteres, esta forma de uso de los sistemas de microblogging ha impulsado las herramientas que permiten acortar las URL para hacerlas mucho más manejables, siendo las más utilizadas, Tinyurl y Bit.ly.

- Ofrecer noticias. En algunas ocasiones creadas y puestas a disposición de los seguidores por proveedores de información y en otros casos son los propios usuarios los que ofrecen noticias y las comentan.

Twitter ofrece un complejo ecosistema de entradas y salidas de información que enlazando con los distintos usos que pueden hacerse de la web colaborativa permiten a los usuarios interactuar con la aplicación desde diferentes puntos de vista (Figura 1). Estas formas de uso de Twitter nos mostrarían a su vez diferentes categorías de usuarios. Entre las más comunes se encontrarían:

- Usuarios que buscan nuevas fuentes de información. Según aumenta el tiempo de uso de Twitter son los propios usuarios los que se convierten a su vez en fuentes de información, tanto originales como amplificando aquellas que consideran útiles para sus seguidores.

- Amigos. En este contexto aparecerían múltiples categorías, desde los amigos creados dentro de Twitter y con los que no hay una relación fuera de Internet, sino que la relación se construye tras el proceso de seguimiento y sucesivos replies o conversaciones, hasta familiares o compañeros añadidos como followers.

La comunicación informal ofrece beneficios que pueden verse desde el punto de vista relacional y desde el ámbito personal. Dentro de los beneficios relacionales, Zhao (2009) considera los siguientes:

- Percepción personal. La percepción personal es un factor importante para la toma de decisiones personales en un entorno de interacción con los otros.

- Lugares comunes. Estos pueden construirse o llegarse a los mismos a través de la conversación. Una conversación informal puede incrementar y aumentar el conocimiento de los puntos de referencia que los usuarios tienen entre sí.

- Conexión. Las conversaciones informales y las conexiones entre participantes permiten aumentar el acceso a las informaciones valiosas

Los beneficios personales estarían relacionados con la puesta en común de intereses y objetivos, publicado de forma libremente en un entorno de rapidez y compartiéndolos con el resto de la comunidad.

El microblogging podría considerarse como una forma de blogging en pequeña escala mediante el uso de los mensajes breves enviados a Internet por individuos y empresas para compartir noticias, actualizaciones de estado y participar en conversaciones. El microblogging está especialmente indicado para hacer rápidas reflexiones, intercambio de ideas y compartir información (Templeton, 2008). Twitter es uno de los primeros ejemplos del microblogging aplicado a la movilidad 2.0 (Grisworld, 2007; Rheinhardt, Ebner, Beham y

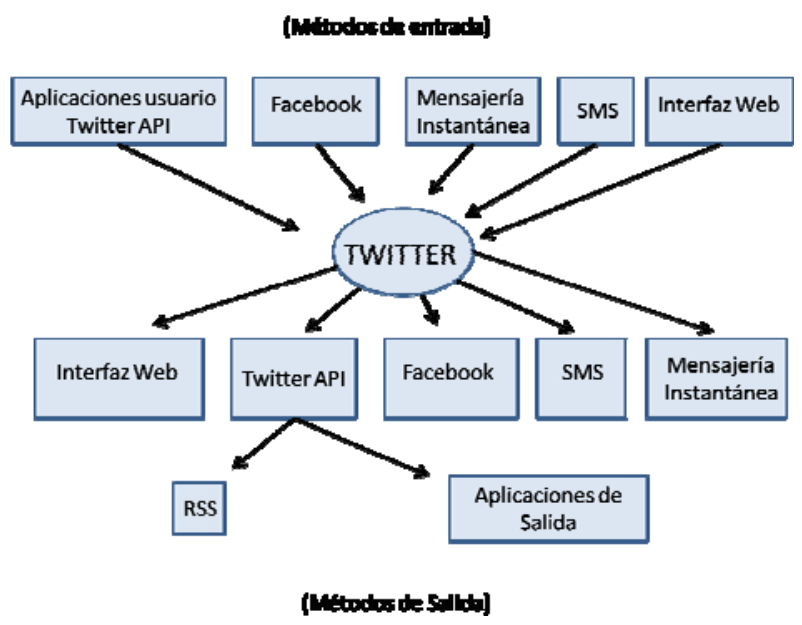

Figura 1. Métodos de entrada y salida de información a través de Twitter (Fuente: elaboración propia) 
Costa, 2009). Gracias a aplicaciones desarrolladas por terceras partes como Twitpic o blip.fm la información compartida puede ser imágenes o música además de textos. Un aspecto interesante que ofrece Twitter es la posibilidad de etiquetar los microposts o tweets. Este etiquetado conforma los denominados hashtags, que es una forma de agrupar los mensajes sobre un determinado tema con el símbolo “\#” seguido de una palabra o código específico. Los hashtags permiten generar una completa base de conocimiento sobre cualquier tema publicado en Twitter.

Otra opción interesante para el uso de Twitter es su utilización antes, durante y después de una conferencia o evento. Los hashtags ayudan a hacer un seguimiento en remoto de lo que está ocurriendo, y cada vez más eventos relacionados con el ámbito de la tecnología ofrecen la posibilidad de ser seguidos a través de los tweets de sus participantes.

Los tweets unidos al etiquetado que los propios participantes añaden se están convirtiendo en un nuevo tipo de meme (Costa et $\mathrm{Al}, 2008$ ). El volumen de respuestas es mucho mayor que las producidas en la conversación a través de un blog, que habitualmente genera una conversación menor de la esperada (Stevens, 2008), además el hecho de que la API con la que está construida Twitter sea pública permite que se generen una gran cantidad de mashups que ofrecen funcionalidades añadidas y que aportan mayor viralidad a su éxito, permitiendo que Twitter no sea simplemente una aplicación de microblogging, sino que sus posibilidades de uso se expandan en función de la imaginación de los desarrolladores.

El contenido generado por los usuarios, uno de los aspectos clave de la Web 2.0, se ha visto reforzado con la popularización de los sistemas de microblogging. Así mismo, tanto las contestaciones con forma de replies como los mensajes directos permiten que la conversación virtual se expanda. No obstante, en mayo de 2009, Twitter realizó ajustes en su plataforma para evitar que los usuarios vieran los replies que hacían sus "amigos" hacia terceros, tratando de evitar de esta manera el "ruido" generado por muchos usuarios que utilizan Twitter como sistema de chat asíncrono. Esta acción fue contestada agriamente por muchos participantes ya que se consideraba una buena forma de conocer a nuevos usuarios.

En estos momentos la mayoría de los mensajes realizados por los usuarios tienen un alto componente de replies magnificando el uso de Twitter como generador de comunidad o red social. Los hashtags o etiquetado de temas apoyan ese sentimiento y desde mayo de 2009 Twitter ofrece en su página de inicio un buscador y muestra los temas más comentados, denominados Trending Topics. Además desde el buscador http://search.twitter.com se puede acceder a cualquier tema que esté siendo publicado por los usuarios y suscribirse al mismo a través de un feed RSS. El buscador fue creado por Summize y adquirido en julio de 2008 por Twitter para mejorar el servicio ante las cada vez más frecuentes críticas de lo complejo que podía resultar encontrar determinada información.

Otra de las prácticas que aumenta el sentimiento de red social es la costumbre de Retweeting, la identificación con las letras RT de un mensaje de un usuario y volver a reproducirlo, de forma que la fórmulaRT@nombredeusuario replica las convenciones sobre los memes que se producen en la blogosfera.

El microblogging ha sido empujado a la popularidad por Twitter permitiendo la aparición de múltiples clones. Tanto por el tipo de los mensajes, ya sean personales o profesionales, como por el enlace a fuentes de referencia, así como por la conversación producida, su funcionamiento es similar a los blogs, pero en este caso en formato micropost. Gracias a la API pública de Twitter se han podido generalizar decenas de aplicaciones que ayudan a mejorar el servicio, programas cliente que permiten acceder a Twitter desde el escritorio del ordenador o el teléfono móvil, crear grupos de usuarios y, en definitiva, mejorar la experiencia de uso. Su API permite también que los desarrolladores hayan construido aplicaciones diseñadas para mostrar Tweets específicos relacionados con el estatus, el público, avisos, la línea temporal de mensajes, las actualizaciones, los seguidores, etc. No obstante, Twitter es una aplicación abierta en término de datos, pero no es Open Source. Su alternativa desarrollada siguiendo las pautas de Open Source es Identi.ca. 


\section{2.- EL USO DEL MICROBLOGGING EN EL ENTORNO EDUCATIVO}

El uso del microblogging en el aula se convierte en la introducción de una herramienta conversacional que lleva los debates fuera del espacio del aula al igual que los blogs, aumentando la sensación de comunidad, mejorando la productividad de las conversaciones, incrementando las interacciones respetuosas entre todos los participantes y finalmente el conocimiento de todos ellos. La observación del Time Line de la herramienta de microblogging puede mostrar algo de ruido o cierta confusión por el elevado número de mensajes publicados, sobre todo según los participantes vayan siguiendo a más personas, pero también ofrece un sentido de variedad e motiva el interés por el descubrimiento de nuevos contactos con intereses similares.

Mediante estos servicios es posible hacer el seguimiento de determinados términos, temas comunes o hashtags, etiquetados habitualmente con el símbolo “\#” y participar libremente en ellos. El microblogging se manifiesta también como un entorno de creación de un cuaderno de notas público, desde el que el docente y el estudiante pueden ir aportando ideas de reflexión y trabajo sobre las que pueda volverse posteriormente para realizar una reflexión más pausada.

Frente a la incorporación de los blogs, más sencilla, ya que suelen ser vistos por los estudiantes como un tipo de página Web en la que de forma sencilla pueden compartir los contenidos que ellos creen convenientes, la metodología de implantación del microblogging en el aula ofrece una mayor complejidad, puesto que muchos de los participantes no conocerán nada de esas aplicaciones, aunque estarán familiarizados con funcionalidades parecidas, para indicar el estatus del usuario en cada momento, puestas en marcha por las redes sociales como Tuenti y Facebook. La metodología de uso ha de contar pues con el desconocimiento y la desconfianza en un primer momento. Por ello es necesario seguir los siguientes procesos:

a) Proveer un contexto de uso

Explicación del por qué se va a utilizar una herramienta que pueden considerar atípica en el contexto de la clase, tanto dentro como fuera del aula.

b) Motivar al registro en la plataforma

No es fácil que los alumnos se registren en una herramienta de estas características por su cuenta, por ello es necesario un trabajo de motivación y apoyo.

c) Inculcar sentido de la responsabilidad

Al igual que la presencia digital de los blogs, el microblogging y la alta visibilidad que se obtiene requiere que los participantes sean conscientes de su uso responsable.

d) Formar sobre las funcionalidades

Para un óptimo uso del sistema es conveniente que los estudiantes sepan utilizar todas las posibilidades de funcionamiento: seguir a otras personas, responder, enviar mensajes directos, hacer búsquedas o participar en debates existentes fuera y dentro de la clase.

e) Identificar a los participantes claramente

Al ser una actividad docente es importante que todos los estudiantes se registren con nombres representativos más que con nicks que sean difíciles de identificar.

f) Seguir y ser seguido

El microblogging tendrá éxito siempre que todos los miembros de la clase se sigan entre sí y al profesor. Para la organización de grandes números de alumnos, lo más conveniente es la creación de grupos, permitidos ya sea por las plataformas o por los programas creados por terceras partes.

Previamente a la puesta en marcha de la estrategia descrita es necesario haber tomado la decisión sobre la herramienta que va a utilizarse entre las actualmente existentes y que han ido 
desarrollándose a lo largo de los últimos años ofreciendo características muy parecidas pero con diferencias sustanciales.

\section{TWITTER COMO HERRAMIENTA DE MICROBLOGGING EDUCATIVO}

El uso de herramientas de microblogging como Twitter no es sencillo para los nuevos usuarios de la Web 2.0 que puedan no sentirse motivados por ello. No obstante puede ser utilizado como herramienta de apoyo. Para su mejor aprovechamiento es importante la utilización de un programa cliente, de forma que los alumnos puedan seguir los replies y los mensajes directos con facilidad. También es positiva la incorporación de herramientas como Twittpic para la publicación de material gráfico, de forma sencilla e intuitiva.

En el ámbito de la docencia Twitter puede incorporar las siguientes opciones (Checa y Joyanes, 2009):

1. Sentido de comunidad. Gracias a él Twitter ha ido creciendo. La capacidad de observar desde los mensajes hasta las fotografías o los perfiles y saber lo que hacen los compañeros en todo momento incrementa el sentido de pertenencia a una red social activa.

2. Conexión permanente. Un sistema de microblogging como Twitter permite trabajar el sentido de formación ubicua y romper el estricto molde de los Learning Management Systems (LMS) tradicionales utilizados en la mayoría de los centros educativos. Desde cualquier ubicación es posible acceder a una plataforma liviana y ágil.

3. Omnipresencia. Mediante el uso activo de herramientas de microblogging el sentimiento de cercanía entre alumnos y con el profesor se ve reforzado.

4. Mejora de experiencia en la publicación de contenidos. Es factible plantear en el espacio de microblogging los mismos objetivos que en la creación de un blog, incrementando el nivel de conversación conseguida.

5. Rapidez para plantear preguntas y respuestas, incrementando la riqueza de las mismas y la facilidad de seguimiento.

6. Indicador de presencia. Las herramientas de microblogging ofrecen información de primera mano de los aspectos relevantes de una conferencia, reunión, clase o momento actual de una investigación.

7. Extrema rapidez en un entorno en el que la agilidad es parte integrante de los participantes, especialmente en la comunidad estudiantil.

8. Generación de conocimiento inesperado. El alumno se hace protagonista de la creación de su propio conocimiento.

9. Aprendizaje informal. El compartir ideas rápidamente y rompiendo los clásicos esquemas de trabajo hace que se cree un aprendizaje en red fuera de las reglas habituales.

10. Mejora de habilidades sociales. El microblogging en general y Twitter en particular actúa como catalizador de la Gestión del Conocimiento, impulsando modelos de colaboración incrementando la sensación de comunidad.

Siguiendo a Tony Wanger de School Change Consulting (2008), es posible reconocer siete competencias básicas para que los estudiantes tengan éxito en la era de la información. Estas serían las siguientes:

- Resolución de problemas y pensamiento crítico

- Colaboración en redes y liderazgo en las mismas

- Agilidad y adaptabilidad

- Iniciativa y espíritu emprendedor

- Dominio del lenguaje oral y escrito

- Acceso a la información y análisis de la misma

- Curiosidad e imaginación 
Gracias a una herramienta como Twitter estas competencias pueden trabajarse e impulsarse. Twitter como herramienta educativa que permite la confluencia entre los mensajes síncronos y asíncronos ofrece una novedad crucial que supera la inicial desconfianza ante la aplicación. La clase continúa después de la sesión en el aula. Los estudiantes comparten públicamente la experiencia de la clase y la comunidad cerrada generada dentro del aula es superada (Lenhart, 2005; Lenhart y Madden, 2007).

Twitter puede ser criticado, tras una breve aproximación al mismo, por la trivialidad aparente de muchos de sus mensajes, pero el auténtico valor es generado por la acumulación. En ese sentido, una de las opciones que se ven reforzadas gracias a su uso docente es la sensación de globalidad. La observación de la línea de tiempo de cada usuario ofrece una alta cantidad de "ruido" pero también muestra variedad y obliga a enfrentarse a conversaciones diferentes. Gracias al uso de Twitter es posible llevar a cabo un seguimiento de palabras o conceptos. Realizar una investigación que lleve a acceder a usuarios desconocidos que estén conversando sobre ese tema.

Mediante su uso en una conferencia es factible acceder a lo que allí está ocurriendo y ofrecer feedback inmediato. Según aumenta el número de seguidores las respuestas ante cualquier cuestión surgen con gran facilidad. A ello se le une la posibilidad de seguir a profesionales, de forma que los estudiantes puedan acceder a profesionales expertos y a personalidades famosas de cada área de estudio.

Twitter es un bloc de notas público, de forma que todos los contenidos quedan disponibles on line, pudiendo ser una buena forma de inspiración volviendo sobre ello más adelante y exigiendo un mayor esfuerzo en la creación de estructuras gramaticales, al estar obligados a ceñirse a un espacio limitado de 140 caracteres, de forma que la transmisión de las ideas ha de hacerse en un espacio reducido. A ello se le suma el que Twitter es utilizado por usuarios que intentan mantener un alto grado de corrección lingüística, siendo muy rechazado la expresión con un elevado uso de abreviaturas o de lenguaje parecido al de los mensajes telefónicos SMS (Huberman, Romero y Wu 2008).

Los estudiantes usuarios de Twitter participan de una tormenta de ideas permanente, pueden comentar procedimientos y proyectos y dialogar sin problemas de barreras físicas o geográficas.

\section{ASPECTOS NEGATIVOS EN EL USO DE TWITTER EN EL AULA}

Además de los aspectos positivos que hemos observado, no debemos olvidar que Twitter también ofrece algunas dificultades o problemas. Por un lado, su uso consume un tiempo elevado, tanto para los alumnos como para los profesores, y genera que las obligaciones clásicas del docente se multipliquen incorporando aspectos ante los que el profesor puede no estar preparado.

Para un correcto uso es necesario el establecimiento de una estrategia previa, ya que sin ella simplemente será un espacio de escritura, en el que puede perderse el sentido final.

Tampoco hemos de olvidar que Twitter, al igual que el resto de herramientas de comunicación mediada, no reemplaza la relación personal y cara a cara, pudiendo generarse situaciones incómodas o mal entendidas ante determinados mensajes. Twitter no llega a reemplazar el correo electrónico y no es previsible que lo haga, ofreciendo este último mayores posibilidades en la transmisión de mensajes complejos.

Finalmente, muchos colegas de profesión pueden no considerar el uso de Twitter como una herramienta académicamente interesante e incluso si es utilizado por las instituciones abre estas a una mayor crítica e incluso a ataques malintencionados de anónimos.

\section{CONCLUSIÓN}

La red es el mayor almacén de recursos disponibles en el plano educativo, pero en muchas ocasiones la relevancia o accesibilidad por parte de los estudiantes no es fácil. El microblogging en general y Twitter en particular pueden cubrir perfectamente los aspectos de comunicación, de recopilación, de organización, de integración o de generación de conocimiento. 
Así mismo, desde el mes de noviembre de 2009 Twitter está disponible en castellano, de forma que aquellos usuarios con problemas con el inglés pueden usarlo sin gran dificultad. Pero es fundamental realizar una planificación de cualquier acción formativa utilizando Twitter para evitar que el alumno se sienta perdido y no sepa muy bien qué es lo que se le exige. Para ello, no podemos olvidar la importancia del aprendizaje informal, pero que este no puede sustituir el reglado o formal.

\section{6.- REFERENCIAS BIBLIOGRÁFICAS}

Checa, F., y Joyanes, L. (2009). Microblogging en el aula. Perspectivas docentes en la aplicación de Twitter como elemento educativo, Congreso Internacional sobre uso y buenas prácticas con TIC: la Web 2.0, Universidad de Málaga. Diciembre.

Cheng, A., y Evans, M. (2009). Inside Twitter An In-Depth Look Inside the Twitter World, SYSOMOS Inc [en línea]. Disponible en http://www.sysomos.com/docs/Inside-Twitter-BySysomos.pdf [Consulta: 2012, 12 de febrero]

Codel, E. (2006). LunchMeet: Twitter is the Obvious Choice [en línea], disponible en: http://www.podtech.net/home/1607/lunchmeet-twitter-is-the-obvious-choice.html [Consulta: 2012, 1 de marzo]

Costa, C. Beham, G., y Rehinhardt, W. (2008). Microblogging In Technology Enhanced Learning: A Use-Case Inspection of PPE Summer School 2008, Workshop at the European Conference on Technology Enhanced Learning (ECTEL), Maastricht

Ebner, M., y Maurer, H. (2009) Can Weblogs and Microblogs Change Traditional Scientific Writing?, Future Internet, [en línea] disponible en www.mdpi.com/journal/futureinternet, 1, 47-58 [Consulta: 2012, 13 de febrero]

Ebner, M., y Schiefner, M. (2008) Microblogging - more than fun?, IADIS Mobile Learning Conference 2008, (pp. 155-159)

Glaser, M. (2007). Twitter founders thrive on micro-blogging constraints [En línea], disponible en http://www.pbs.org/mediashift/2007/05/digging_deepertwitter_founders.html [Consulta: 2012, 1 de marzo]

Griswold, W.G. (2007). Five Enablers for Mobile 2.0, Computer, Universidad de California, San Diego; (pp. 96-98), San Diego: IEEE

Huberman, B., Romero, D., y Wu, F. (2008). Social networks that matter: Twitter under the microscope, First Monday, 14(1) [en línea], disponible en http://firstmonday.org/htbin/cgiwrap/bin/ojs/index.php/fm/article/view/2317/2063 [Consulta: 2011, 18 de febrero]

Java, A., Finim, T., Song, X., y Tseng, B. (2007). Why we twitter: understanding microblogging usage and communities, International Conference on Knowledge Discovery and Data Mining (pp. 56-65). Nueva York: ACM.

Krisnamurthy, B., Gill, P., y Arlitt, M. (2008). A few chirps about twitter, First workshop on Online social networks: The face of social networks; (pp. 19-24). Seattle: ACM

Lenhart, A, (2005). “Teen content creators and consumers," Pew Internet \& American Life Project.

Lenhart, A. Madden, M. (2007) Social Networking Websites and Teens: An Overview, Pew Internet \& American Life Project

Milstein, S., Choudhury, A., Hochmuth, G., Lorica, B., y Magoulas, R. (2008) .Twitter and the Micro-Messaging Revolution: Communication, Connections, and Immediacy--140 Characters at a Time, O'Reilly Radar Report, Noviembre

Mischaud, E. (2007). Twitter: expressions of the whole self, London School of Economics LSE - MEDIA@LSE. [En línea], disponible en http://www.lse.ac.uk/collections/media@lse/mediaWorkingPapers/MScDissertationSeries/Mishaud_Fin al.pdf [Consulta: 2012, 3 de marzo]

Reinhardt, W. Ebner, M., Beham, G., y Costa, C. (2009). How People are using Twitter during Conferences, Creativity and Innovation Competencies on the Web, 5th. EduMedia Conference, (pp. 145-156), Salzburgo: Hornung-Prähauser

Stevens, V. (2008). Trial by Twitter: The Rise and Slide of the Year's Most Viral Microblogging Platform, TESL-EJ, 12(1) [en línea], disponible en http://tesl-ej.org/ej45/int.html [Consulta: 2012, 1 de junio] 
Templeton, M. (2008). Microblogging Defined [en línea]. Disponible en http://microblink.com/2008/11/11/microblogging-defined/ [Consulta: 2012, 1 de marzo]

Zhao, D. (2009). "How and why people Twitter: the role that micro-blogging plays in informal communication at work", Conference on Supporting Group Work (pp. 243-252). Nueva York: ACM

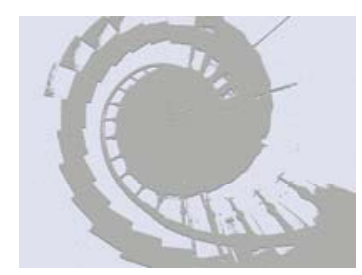

